\title{
QUASI-ARMENDARIZ PROPERTY ON POWERS OF COEFFICIENTS
}

Tai Keun Kwak, Min Jung Lee and Yang Lee

Received: 30 October 2013

Communicated by Pınar Aydoğdu

Dedicated to the memory of Professor Efraim P. Armendariz

\begin{abstract}
The study of Armendariz rings was initiated by Rege and Chhawchharia, based on a result of Armendariz related to the structure of reduced rings. Armendariz rings were generalized to quasi-Armendariz rings by $\mathrm{Hi}$ rano. We introduce the concept of power-quasi-Armendariz (simply, p.q.Armendariz) ring as a generalization of quasi-Armendariz, applying the role of quasi-Armendariz on the powers of coefficients of zero-dividing polynomials. In the process we investigate the power-quasi-Armendariz property of several ring extensions, e.g., matrix rings and polynomial rings, which have roles in ring theory.
\end{abstract}

Mathematics Subject Classification (2010): $16 \mathrm{U} 80$

Keywords: power-quasi-Armendariz ring, power of coefficient, quasi-Armendariz ring, Armendariz ring, polynomial ring, matrix ring

\section{Introduction}

Throughout this note every ring is associative with identity unless otherwise specified. Given a ring $R, J(R), N^{*}(R)$ and $N(R)$ denote the Jacobson radical, the upper nilradical (i.e., sum of all nil ideals) and the set of all nilpotent elements in $R$, respectively. It is well-known that $N^{*}(R) \subseteq J(R)$ and $N^{*}(R) \subseteq N(R)$. We use $R[x]$ to denote the polynomial ring with an indeterminate $x$ over given a ring $R$. For $f(x) \in R[x]$, let $C_{f(x)}$ denote the set of all coefficients of $f(x)$. $\mathbb{Z}$ (resp., $\mathbb{Z}_{n}$ ) denotes the ring of integers (resp., the ring of integers modulo $n$ ). Denote the $n$ by $n$ full (resp., upper triangular) matrix ring over a ring $R$ by $\operatorname{Mat}_{n}(R)$ (resp.,

The first named author was supported by Basic Science Research Program through the National Research Foundation of Korea(NRF) funded by the Ministry of Education, Science and Technology(No. 2010-0022160). The third named author was supported by Basic Science Research Program through the National Research Foundation of Korea(NRF) funded by the Ministry of Education(NRF-2013R1A1A2A10004687). 
$\left.U_{n}(R)\right)$ for $n \geq 2$. Next let

$D_{n}(R)$ be the subring $\left\{m \in U_{n}(R) \mid\right.$ the diagonal entries of $m$ are all equal $\}$

of $U_{n}(R)$

$$
N_{n}(R)=\left\{\left(a_{i j}\right) \in D_{n}(R) \mid a_{i i}=0 \text { for all } i\right\}, \text { and }
$$

$V_{n}(R)=\left\{\left(a_{i j}\right) \in D_{n}(R) \mid a_{i j}=a_{(i+1)(j+1)}\right.$ for $i=1, \ldots, n-2$ and $\left.j=2, \ldots, n-1\right\}$.

Note that $V_{n}(R) \cong R[x] /\left(x^{n}\right)$, where $\left(x^{n}\right)$ is the ideal of $R[x]$ generated by $x^{n}$. Use $E_{i j}$ for the matrix with $(i, j)$-entry 1 and other entries 0 .

For a ring $R$ and an $(R, R)$-bimodule $M$, the trivial extension of $R$ by $M$ is the ring $T(R, M)=R \oplus M$ with the usual addition and the following multiplication: $\left(r_{1}, m_{1}\right)\left(r_{2}, m_{2}\right)=\left(r_{1} r_{2}, r_{1} m_{2}+m_{1} r_{2}\right)$. This is isomorphic to the ring of all matrices $\left(\begin{array}{cc}r & m \\ 0 & r\end{array}\right)$, where $r \in R$ and $m \in M$ and the usual matrix operations are used.

A ring is called reduced if it has no nonzero nilpotent elements. Rege and Chhawchharia [15] called a ring $R$ (not necessarily with identity) Armendariz if $a b=0$ for all $a \in C_{f(x)}$ and $b \in C_{g(x)}$ whenever $f(x) g(x)=0$ for $f(x), g(x) \in R[x]$ based on [2, Lemma 1]. Reduced rings are clearly Armendariz. A ring is usually called Abelian if every idempotent is central. Armendariz rings are Abelian by [10, Lemma 7]. The concept of Armendariz ring was generalized to the quasiArmendariz ring property by Hirano. A ring $R$ (not necessarily with identity) is called quasi-Armendariz [7] provided that

$$
a R b=0 \text { for all } a \in C_{f(x)} \text { and } b \in C_{g(x)} \text { whenever } f(x) R g(x)=0
$$

for $f(x), g(x) \in R[x]$.

Semiprime rings are quasi-Armendariz rings by [7, Corollary 3.8], but not conversely in general.

On the other hand, Han et al. [6] called a ring $R$ (not necessarily with identity) power-Armendariz if whenever $f(x) g(x)=0$ for $f(x), g(x) \in R[x]$, there exist $m, n \geq 1$ such that

$$
a^{m} b^{n}=0 \text { for all } a \in C_{f(x)}, b \in C_{g(x)}
$$

The class of quasi-Armendariz rings and the class of power-Armendariz rings do not imply each other by Example 2.1 to follow. 


\section{Power-quasi-Armendariz rings}

We first consider the following condition $(\dagger)$ : There exist $m, n \geq 1$ such that

$$
a^{m} R b^{n}=0 \text { for any } a \in C_{f(x)} \text { and } b \in C_{g(x)} \text {, whenever } f(x) R g(x)=0
$$

for $f(x), g(x) \in R[x]$, where $R$ is a ring, not necessarily with identity.

It is obvious that $a^{m} R b^{n}=0$ for some $m, n \geq 1$ if and only if $a^{\ell} R b^{\ell}=0$ for some $\ell \geq 1$, in the condition $(\dagger)$ above. Quasi-Armendariz rings clearly satisfy the condition $(\dagger)$, but each part of the following example shows that the class of rings satisfying the condition $(\dagger)$ need not be quasi-Armendariz or power-Armendariz.

Example 2.1. (1) Consider a ring $R=D_{n}(T)$ where $T=T(W, W)$ for a division ring $W$ and $n \geq 2$. Let $f(x)=\sum_{i=0}^{s} A_{i} x^{i}, g(x)=\sum_{j=0}^{t} B_{j} x^{j} \in R[x]$ with $f(x) R g(x)=0$. Since $J(R)=N_{n}(T)$ and $\frac{R}{N_{n}(T)} \cong T, f(x) R g(x)=0$ implies that $A_{i}, B_{j} \in N_{n}(T)$ for all $i, j$. Then $A_{i}^{n}=0=B_{j}^{n}$ and so $A_{i}^{n} R B_{j}^{n}=0$, showing that $R$ satisfies the condition ( $\dagger$ ). However, $R$ is not quasi-Armendariz by help of $[3$, Example 2.5]. Note that $R$ is power-Armendariz.

(2) Consider a ring $R=\operatorname{Mat}_{n}(A)$ where $A$ is a quasi-Armendariz ring and $n \geq 2$. Then $R$ is quasi-Armendariz by [7, Theorem 3.12] and so it satisfies the condition ( $\dagger$ ), but not power-Armendariz by [6, Example 1.5(1)].

Based on the above, we will call a ring $R$ (not necessarily with identity) powerquasi-Armendariz (shortly, p.q.-Armendariz) if it satisfies the condition $(\dagger)$. Hence, the concept of p.q.-Armendariz ring is a generalization of a quasi-Armendariz ring.

Due to Lambek [13], an ideal $I$ of a ring $R$ is called symmetric if $a b c \in I$ implies $a c b \in I$ for all $a, b, c \in R$. If the zero ideal of a $\operatorname{ring} R$ is symmetric then $R$ is called symmetric. Following Bell [4], a ring $R$ is called to satisfy the Insertion-ofFactors-Property (simply, an IFP ring) if $a b=0$ implies $a R b=0$ for $a, b \in R$. Note that $N(R)=N^{*}(R)$ for an IFP ring $R$ by [16, Theorem 1.5]. Reduced rings are symmetric and symmetric rings are IFP, and a simple computation yields that IFP rings are Abelian. We see that $D_{3}(R)$ is an IFP ring and $D_{n}(R)$ is not IFP for $n \geq 4$ in [11], where $R$ is a reduced ring.

Recall that a ring $R$ is called almost symmetric [16] if $R$ is IFP and satisfies the following condition:

(S II) $a b^{m} c^{m}=0$ for some positive integer $m$ whenever $a(b c)^{n}=0$

for given $n \geq 1$ and $a, b, c \in R$. 
Symmetric rings are almost symmetric, but not conversely by [16, Proposition 1.4 and Example 5.1], and almost symmetric rings are obviously IFP, however the class of IFP rings and the class of rings satisfying the condition (S II) are independent of each other by [16, Example 5.1(c) and Example 5.2(b)]. Symmetric rings are power-Armendariz by [6, Proposition 1.1(4)].

Proposition 2.2. (1) If $R$ is a p.q.-Armendariz ring, then so is eRe for $0 \neq e^{2}=$ $e \in R$.

(2) The class of p.q.-Armendariz rings is closed under direct sum.

(3) Almost symmetric rings are p.q.-Armendariz.

(4) Power-Armendariz IFP rings are p.q.-Armendariz.

Proof. (1) Let $f(x), g(x) \in e R e[x]$ such that $f(x)(e R e) g(x)=0$. Since $f(x) e=$ $f(x)$ and $e g(x)=g(x)$, we have $f(x) R g(x)=0$. Assume that $R$ is p.q.-Armendariz. Then there exist $m, n \geq 1$ such $a^{m} R b^{n}=0$ for any $a \in C_{f(x)}, b \in C_{g(x)}$. Since $a=a e$ and $b=e b, 0=a^{m} R b^{n}=\underbrace{a \cdots a}_{m-1} a e R e b \underbrace{b \cdots b}_{n-1}=a^{m}(e R e) b^{n}$ and thus $e R e$ is p.q.-Armendariz.

(2) Let $R_{u}$ be p.q.-Armendariz rings for all $u \in U$ and $E=\oplus_{u \in U} R_{u}$, the direct sum of $R_{u}$ 's. Suppose that $f(x) E g(x)=0$ for $0 \neq f(x)=\sum_{i=0}^{s}\left(a(i)_{u}\right) x^{i}, 0 \neq$ $g(x)=\sum_{j=0}^{t}\left(b(j)_{u}\right) x^{j} \in E[x]$. We apply the proof of [6, Proposition 1.1(1)]. Note that $f(x)$ and $g(x)$ can be rewritten by

$$
f(x)=\left(\sum_{i=0}^{s} a(i)_{u} x^{i}\right), g(x)=\left(\sum_{j=0}^{t} b(j)_{u} x^{j}\right) \in \oplus_{u \in U} R_{u}[x] .
$$

$f(x) E g(x)=0$ yields $\left(\sum_{i=0}^{s} a(i)_{u} x^{i}\right) E\left(\sum_{j=0}^{t} b(j)_{u} x^{j}\right)=0$ for all $u \in U$. Note that finitely many polynomials in $\left\{\left(\sum_{i=0}^{s} a(i)_{u} x^{i}\right),\left(\sum_{j=0}^{t} b(j)_{u} x^{j}\right) \mid u \in U\right\}$ are nonzero. Since $R_{u}$ is p.q.-Armendariz for all $u \in U$. Then there exists $h \geq 1$ such that $\left[a(i)_{u}\right]^{h}\left[b(j)_{u}\right]^{h}=0$ for all $i, j, u$. This implies that $\left(a(i)_{u}\right)^{h} E\left(b(j)_{u}\right)^{h}=0$ for all $i, j$, showing that $E$ is p.q.-Armendariz.

(3) Let $R$ be an almost symmetric ring. Then $N(R)=N^{*}(R)$. Suppose that $f(x) R g(x)=0$ for $f(x)=\sum a_{i} x^{i}, g(x)=\sum b_{j} x^{j} \in R[x]$. We use $\bar{R}$ and $\bar{r}$ to denote $R / N(R)$ and $r+N(R)$, respectively. Since $R / N(R)$ is reduced (hence quasiArmendariz) and $\left(\sum \bar{a}_{i} x^{i}\right) \bar{R}\left(\sum \bar{b}_{j} x^{j}\right)=0$, we have $a R b \subseteq N(R)$ for any $a \in C_{f(x)}$ and $b \in C_{g(x)}$. Then $(a R b)^{n}=0$ and so $(a b)^{n}=0$ for some $n \geq 1$. Since $R$ is almost symmetric, $a^{l} b^{l}=0$ and so $a^{l} R b^{l}=0$ for some $l \geq 1$. Thus $R$ is p.q.-Armendariz.

(4) is simply checked through a simple computation. 
Corollary 2.3. Let e be a central idempotent of a ring $R$. Then $R$ is p.q.Armendariz if and only if $e R$ and $(1-e) R$ are both p.q.-Armendariz.

Proof. It follows from Proposition 2.2(1,2), since $R \cong e R \oplus(1-e) R$.

Example 2.4. The ring $R=U_{2}(D)$ for a domain $D$ is quasi-Armendariz by $[7$, Corollary 3.15] and hence $R$ is p.q.-Armendariz, but not IFP.

Proposition 2.5. Let $R$ be a ring and $I$ be a proper ideal of $R$. If $R / I$ is a p.q.-Armendariz ring and $I$ is reduced as a ring without identity, then $R$ is p.q.Armendariz.

Proof. We adapt the proof of [6, Theorem 1.11(4)]. Let $f(x) R g(x)=0$ for $f(x), g(x) \in R[x]$. Since $R / I$ is p.q.-Armendariz, there exists $s \geq 1$ such that $a^{s} R b^{s} \subseteq I$ for any $a \in C_{f(x)}$ and $b \in C_{g(x)}$. By the same computation as in the proof of [6, Theorem 1.11(4)], we have $a I b=0$ for any $a \in C_{f(x)}$ and $b \in C_{g(x)}$, and thus

$$
a^{s+1} R b^{s+1}=a\left(a^{s} R b^{s}\right) b \in a I b,
$$

and hence $a^{s+1} R b^{s+1}=0$. Therefore $R$ is p.q.-Armendariz.

Proposition 2.6. For a ring $R$, if $\mathrm{Mat}_{n}(R)$ (resp., $U_{n}(R)$ ) is p.q-Armendariz for $n \geq 2$, then $R$ is p.q.-Armendariz.

Proof. If $\operatorname{Mat}_{n}(R)$ is p.q.-Armendariz, then $R \cong E_{11} \operatorname{Mat}_{n}(R) E_{11}$ is p.q.-Armendariz by Proposition 2.2(1).

We actually do not know whether $\operatorname{Mat}_{n}(R)$ (resp., $U_{n}(R)$ ) is p.q.-Armendariz if $R$ is a p.q.-Armendariz ring.

Question. If $R$ is a p.q.-Armendariz ring, then is $\operatorname{Mat}_{n}(R)$ (resp., $U_{n}(R)$ ) p.q.Armendariz?

But we find the following kinds of subrings of $\operatorname{Mat}_{n}(R)$ which preserve the p.q. Armendariz property.

Theorem 2.7. Let $R$ be an IFP ring and $n \geq 2$. The following conditions are equivalent:

(1) $R$ is p.q.-Armendariz.

(2) $D_{n}(R)$ is p.q.-Armendariz.

(3) $V_{n}(R)$ is p.q.-Armendariz.

(4) $T(R, R)$ is p.q.-Armendariz. 
Proof. (1) $\Rightarrow(2)$ : Let $f(x)=\sum_{i=0}^{s} A_{i} x^{i}, g(x)=\sum_{j=0}^{t} B_{j} x^{j} \in D_{n}(R)[x]$ satisfy $f(x) D_{n}(R) g(x)=0$, where $A_{i}=\left(a(i)_{c d}\right)$ and $B_{j}=\left(b(j)_{h k}\right)$ for $0 \leq i \leq s$ and $0 \leq j \leq t$. The proof is similar to one of [6, Theorem 1.4(1)], but we write it here for completeness.

Note that $f(x)$ and $g(x)$ can be expressed by

$$
f(x)=\left(\begin{array}{ccccc}
f_{11}(x) & f_{12}(x) & f_{13}(x) & \cdots & f_{1 n}(x) \\
0 & f_{22}(x) & f_{23}(x) & \cdots & f_{2 n}(x) \\
0 & 0 & f_{33}(x) & \cdots & f_{3 n}(x) \\
\vdots & \vdots & \vdots & \ddots & \vdots \\
0 & 0 & 0 & \cdots & f_{n n}(x)
\end{array}\right)
$$

and

$$
g(x)=\left(\begin{array}{ccccc}
g_{11}(x) & g_{12}(x) & g_{13}(x) & \cdots & g_{1 n}(x) \\
0 & g_{22}(x) & g_{23}(x) & \cdots & g_{2 n}(x) \\
0 & 0 & g_{33}(x) & \cdots & g_{3 n}(x) \\
\vdots & \vdots & \vdots & \ddots & \vdots \\
0 & 0 & 0 & \cdots & g_{n n}(x)
\end{array}\right)
$$

where

$$
f_{11}(x)=\cdots=f_{n n}(x)=\sum_{i=0}^{s} a(i)_{11} x^{i}, f_{c d}(x)=\sum_{i=0}^{s} a(i)_{c d} x^{i}
$$

and

$$
g_{11}(x)=\cdots=g_{n n}(x)=\sum_{j=0}^{t} b(j)_{11} x^{j}, g_{h k}(x)=\sum_{j=0}^{t} b(j)_{h k} x^{j} .
$$

Since $f(x) D_{n}(R) g(x)=0, f_{11}(x) R g_{11}(x)=0$ and so there exist $w \geq 1$ such that $a(i)_{11}^{w} R b(j)_{11}^{w}=0$ for all $i, j$ since $R$ is p.q.-Armendariz.

Next note that every sum-factor of each entry of $A_{i}^{w n}$ (resp., $B_{j}^{w n}$ ) contains $a(i)_{11}^{w}$ (resp., $b(j)_{11}^{w}$ ) in its product by [9, Lemma 1.2(1)]. Now since $R$ is IFP, we get $A_{i}^{w n} R B_{j}^{w n}=0$ because every sum-factor in each entry of $A_{i}^{w n} R B_{j}^{w n}$ is of the form

$$
s a(i)_{11}^{w} t b(j)_{11}^{w} u=0,
$$

for any $s, t, u \in R$.

$(2) \Rightarrow(1)$ : Suppose that $D_{n}(R)$ is p.q.-Armendariz. Let $f(x) R g(x)=0$ for $f(x), g(x) \in R[x]$. Then

$$
\left(f(x) \sum_{i=1}^{n} E_{i i}\right) D_{n}(R)[x]\left(g(x) \sum_{i=1}^{n} E_{i i}\right)=0 .
$$


Since $D_{n}(R)$ is p.q.-Armendariz, there exist $s, t \geq 1$

$$
\left(a \sum_{i=1}^{n} E_{i i}\right)^{s} D_{n}(R)\left(b \sum_{i=1}^{n} E_{i i}\right)^{t}=0
$$

for any $a \in C_{f(x)}$ and $b \in C_{g(x)}$. In particular, for any $r \in R$, we get

$$
\left(a \sum_{i=1}^{n} E_{i i}\right)^{s}\left(r \sum_{i=1}^{n} E_{i i}\right)\left(b \sum_{i=1}^{n} E_{i i}\right)^{t}=0
$$

implying that $a^{s} R b^{t}=0$ for any $a \in C_{f(x)}$ and $b \in C_{g(x)}$. Therefore $R$ is p.q.Armendariz.

$(1) \Leftrightarrow(3)$ and $(1) \Leftrightarrow(4)$ can be obtained by the same argument as in the proof of $(1) \Leftrightarrow(2)$.

The following result comes from Theorem 2.7 and Proposition 2.2(3).

Corollary 2.8. If $R$ is an almost symmetric ring, then $D_{n}(R)$ is p.q.-Armendariz for any $n \geq 2$.

Recall that a ring $R$ is called directly finite if $b a=1$ whenever $a b=1$ for $a, b \in R$. Abelian rings are directly finite and power-Armendariz rings are Abelian by [6, Proposition 1.1(5)]. However, there exists a p.q.-Armendariz ring which is not directly finite (hence non-Abelian) by the following.

Example 2.9. There exists a domain (hence p.q.-Armendariz) $D$ such that $R=$ $\operatorname{Mat}_{2}(D)$ is not directly finite by [14, Theorem 1.0]. Then $R$ is quasi-Armendariz by [7, Theorem 3.12], and so it is p.q.-Armendariz. But $R$ is non-Abelian obviously.

A ring $R$ is called (von Neumann) regular if for each $a \in R$ there exists $b \in R$ such that $a=a b a$. in [5]. Notice that a regular $\operatorname{ring} R$ is power-Armendariz if and only if $R$ is Armendariz if and only if $R$ is Abelian if and only if $R$ is reduced by help of [6, Theorem 1.8]. However, there exists a von Neumann regular p.q.-Armendariz ring but not reduced, by considering $\operatorname{Mat}_{2}(D)$ with $D$ a division ring in Example 2.9 .

Theorem 2.10. (1) If $R[x]$ is a p.q.-Armendariz ring, then so is $R$.

(2) Let $R$ be an IFP ring. If $R$ is p.q.-Armendariz, then so is $R[x]$.

Proof. (1) Suppose that $R[x]$ is a p.q.-Armendariz ring. Let $f(x) R g(x)=0$ for $f(x), g(x) \in R[x]$. Let $y$ be an indeterminate over $R[x]$. Then $f(y) R g(y)=0$ and so $f(y) R[x] g(y)=0$ for $f(y), g(y) \in R[x][y]$, since $x$ commutes with $y$. By hypothesis, there exist $s, t \geq 1$ such that $a^{s} R[x] b^{t}=0$ for any $a \in C_{f(y)}$ and 
$b \in C_{g(y)}$. This implies that $a^{s} R b^{t}=0$ for any $a \in C_{f(x)}$ and $b \in C_{g(x)}$, and thus $R$ is p.q.-Armendariz.

(2) We apply the proof of [6, Proposition 2.2] which was done by help of Anderson and Camillo [1, Theorem 2]. Suppose that $R$ is a p.q-Armendariz IFP ring. Let $p(y)=\sum_{i=0}^{m} f_{i}(x) y^{i}$ and $q(y)=\sum_{j=0}^{n} g_{j}(x) y^{j} \in(R[x])[y]$ with $p(y) R[x] q(y)=0$. Next let $f_{i}(x)=a_{i_{0}}+a_{i_{1}} x+\cdots+a_{i_{w}} x^{i_{w}}, g_{j}(x)=b_{j_{0}}+b_{j_{1}} x+\cdots+b_{j_{v}} x^{j_{v}}$ for each $i, j$, where $a_{i_{0}}, \ldots, a_{i_{w}}, b_{j_{0}}, \ldots, b_{j_{v}} \in R$. Let $k=\sum_{i=0}^{m} \operatorname{deg}\left(f_{i}(x)\right)+\sum_{j=0}^{n} \operatorname{deg}\left(g_{j}(x)\right)$, where the degree is considered as polynomials in $R[x]$ and the degree of zero polynomial is taken to be 0 . Let $p\left(x^{k}\right)=\sum_{i=0}^{m} f_{i}(x)\left(x^{k}\right)^{i}$ and $q\left(x^{k}\right)=\sum_{j=0}^{n} g_{j}(x)\left(x^{k}\right)^{j} \in$ $R[x]$. Then the set of coefficients of the $f_{i}$ 's (resp., $g_{j}$ 's) equals the set of coefficients of $p\left(x^{k}\right)$ (resp., $q\left(x^{k}\right)$ ). From $p(y) R[x] q(y)=0$, we have $p(y) R q(y)=0$ and so $p\left(x^{k}\right) R q\left(x^{k}\right)=0$. Since $R$ is p.q.-Armendariz, there exists $v \geq 1$ such that

$$
a_{\alpha}^{v} R b_{\beta}^{v}=0 \text { for all } \alpha, \beta .
$$

Since $R$ is IFP, we also have

$$
a_{\alpha} R_{1} a_{\alpha} R_{2} \cdots R_{v-1} a_{\alpha} R_{v} b_{\beta} R_{v+1} b_{\beta} R_{v+2} \cdots R_{2 v-1} b_{\beta}=0,
$$

where $R_{1}=\ldots=R_{2 v-1}=R$. Note that some $a_{\alpha^{\prime}}$ (resp., some $b_{\beta^{\prime}}$ ) occurs at least $v$-times (resp., $v$-times) in the coefficient of each monomial in

$$
f_{i}(x)^{(m+1) v}\left(\operatorname{resp} ., g_{j}(x)^{(n+1) v}\right) .
$$

From this we have

$$
f_{i}(x)^{(m+1) v} R g_{j}(x)^{(n+1) v}=0
$$

by the equality (1). This implies that $R[x]$ is p.q.-Armendariz.

Recall that a ring $R$ is called strongly IFP [12] if $R[x]$ is IFP, equivalently, whenever polynomials $f(x), g(x)$ in $R[x]$ satisfy $f(x) g(x)=0, f(x) R g(x)=0$. Clearly strongly IFP rings are IFP, but not conversely by [8, Example 2].

Let $R$ be a strongly IFP ring. Then the Armendariz ring property coincides with the quasi-Armendariz ring property by [12, Proposition 3.18]. This yields the following equivalent conditions by help of [1, Theorem 2] and the fact that the quasi-Armendariz property is closed under subrings:

(1) $R$ is quasi-Armendariz;

(2) $R$ is Armendariz;

(3) $R[x]$ is Armendariz;

(4) $R[x]$ is quasi-Armendariz. 
Acknowledgement. The authors thank the referee for his/her very careful reading of the manuscript and valuable suggestions that improved the paper.

\section{References}

[1] D.D. Anderson and V. Camillo, Armendariz rings and Gaussian rings, Comm. Algebra, 26 (1998), 2265-2272.

[2] E.P. Armendariz, A note on extensions of Baer and P.P.-rings, J. Aust. Math. Soc., 18 (1974), 470-473.

[3] M. Başer, F. Kaynarca, T.K. Kwak and Y. Lee, Weak quasi-Armendariz rings, Algebra Colloq., 18 (2011), 541-552.

[4] H.E. Bell, Near-rings in which each element is a power of itself, Bull. Aust. Math. Soc., 2 (1970), 363-368.

[5] K.R. Goodearl, Von Neumann Regular Rings, Pitman, London, 1979.

[6] J.C. Han, T.K. Kwak, M.J. Lee, Y. Lee and Y.S. Seo, On powers of coefficients of zero-dividing polynomials, submitted.

[7] Y. Hirano, On annihilator ideals of a polynomial ring over a noncommutative ring, J. Pure Appl. Algebra, 168 (2002), 45-52.

[8] C. Huh, Y. Lee and A. Smoktunowicz, Armendariz rings and semicommutative rings, Comm. Algebra, 30 (2002), 751-761.

[9] D.W. Jung, T.K. Kwak, M.J. Lee and Y. Lee, Ring properties related to symmetric rings, submitted.

[10] N.K. Kim and Y. Lee, Armendariz rings and reduced rings, J. Algebra, 223 (2000), 477-488.

[11] N.K. Kim and Y.Lee, Extension of reversible rings, J. Pure Appl. Algebra, 185 (2003), 207-223.

[12] T.K. Kwak, Y. Lee and S.J. Yun, The Armendariz property on ideals, J. Algebra, 354 (2012), 121-135.

[13] J. Lambek, On the representation of modules by sheaves of factor modules, Canad. Math. Bull., 14 (1971), 359-368.

[14] J.C. Shepherdson, Inverses and zero-divisors in matrix ring, Proc. London Math. Soc., 3 (1951), 71-85.

[15] M.B. Rege and S. Chhawchharia, Armendariz rings, Proc. Japan Acad. Ser. A Math. Sci., 73 (1997), 14-17.

[16] G. Shin, Prime ideals and sheaf representation of a pseudo symmetric ring, Trans. Amer. Math. Soc., 184 (1973), 43-60. 


\section{Tai Keun Kwak}

Department of Mathematics

Daejin University

Pocheon 487-711, Korea

e-mail: tkkwak@daejin.ac.kr

Min Jung Lee and Yang Lee

Department of Mathematics Education

Pusan National University

Pusan 609-735, Korea

e-mails: nice1mj@nate.com (Min Jung Lee)

ylee@pusan.ac.kr (Yang Lee) 